\title{
Differentiating Early and Late Recurrences After Resection of HCC in Cirrhotic Patients: Implications on Surveillance, Prevention, and Treatment Strategies
}

\author{
Ronnie T. P. Poon, MBBS, MS, PhD, FRCS (Ed), FACS \\ Department of Surgery, University of Hong Kong Medical Centre, Queen Mary Hospital, Hong Kong, China
}

Hepatocellular carcinoma (HCC) is one of the most common human malignancies. The majority of HCCs occur in a background of cirrhosis related to hepatitis B virus, hepatitis $\mathrm{C}$ virus, or alcoholism. The presence of cirrhosis has significant impact on the management of the cancer. The impairment of liver function as a result of cirrhosis restricts the treatment options, and cirrhosis is also known to predispose to multicentric hepatocarcinogenesis and increase the risk of recurrence after resection of HCC. ${ }^{1}$ The advent of liver transplantation and radiofrequency ablation have provided better chances of curative treatment for patients with small HCC detected by screening in recent years; however, hepatic resection remains the mainstay of curative treatment for HCC associated with Child-Pugh class A cirrhosis. ${ }^{2}$ While the perioperative safety and long-term survival of liver resection in cirrhotic liver have improved in recent years, the rate of postoperative recurrence of $\mathrm{HCC}$ remains high. ${ }^{3}$

In more than $80 \%$ of the cases of postoperative recurrence after resection of HCC, the recurrent tumors occur in the liver remnant. ${ }^{1}$ The mechanisms of intrahepatic recurrence can be either intrahepatic metastasis from the initial tumor or a de novo multicentric tumor. Differentiation of the two has potential implications on surveillance, prevention, and management strategies for recurrence. Clinically, it is impossible to distinguish the two based on imaging appearances. The only definitive way of differentiation is by genetic or molecular studies of the clonal origin of the tumors, which are technically complicated and cannot be used in clinical practice. ${ }^{4,5}$ Hence, several groups

(c) Society of Surgical Oncology 2009

Published Online: 4 February 2009

R. T. P. Poon, MBBS, MS, PhD, FRCS (Ed), FACS

e-mail: poontp@hkucc.hku.hk of authors have attempted to differentiate intrahepatic metastasis and multicentric recurrence based on the interval of development of recurrence from the time of resection and risk factors associated with the recurrence. ${ }^{6-8}$ These studies have consistently demonstrated that early recurrence within the first 2 years after resection of HCC is likely to be associated with aggressive tumor pathological factors such as high tumor grade, microvascular invasion, and microsatellite lesions, whereas late recurrence is more likely related to underlying liver conditions such as the presence of cirrhosis and hepatitis activity. Such results suggested that early recurrence is most likely the consequence of occult metastasis from the initial tumor, whereas late recurrence more likely represents multicentric tumors.

Cucchetti et al. ${ }^{9}$ conducted a study on the risk factors associated with early and late recurrence in 204 patients with cirrhosis who had undergone resection of HCC. The authors identified high alpha-fetoprotein level, poorly differentiated tumor, and microvascular invasion as the risk factors for early recurrence within 2 years after resection, and male gender, older age, high transaminase levels, multiple primary tumors, and high alpha-fetoprotein level as risk factors for late recurrence beyond 2 years. These findings are generally in line with those of previous studies. $^{6-8}$ To demonstrate that late recurrence is most likely related to new multicentric tumor development in the cirrhotic liver, the authors further compared the incidence and risk factors of late recurrence in resected patients with the those of development of HCC in a cohort of 150 cirrhotic patients undergoing regular surveillance of HCC. Such data are not available in previous studies and provide a better insight into the risk of late recurrence after resection of HCC in cirrhotic patients. The authors showed that the risk factors for development of HCC in a group of cirrhotic patients undergoing surveillance were similar to the risk 
factors for late recurrence after resection of HCC in cirrhotic patients. Interestingly, after stratification by risk factors, the annual incidence of recurrence $(6.2 \%$ per year) in low-risk $(<2$ risk factors) resected patients was similar to the annual incidence of HCC (5.8\% per year) in the cirrhotic patients with high risk profile ( $\geq 2$ risk factors). The risk of development of HCC was the highest in the resected group with $\geq 2$ risk factors ( $26.7 \%$ per year) and lowest in cirrhotic patients with $<2$ risk factors. The data suggested that while cirrhotic patients with resection of HCC and those with cirrhosis had similar risk factors for development of new HCC, the former group was at a higher risk of further developing new tumors with the already manifested HCC.

The new findings of the study of Cucchetti et al. ${ }^{9}$ may provide guidance on the optimum surveillance strategy after resection of HCC. Currently, the development of recurrence after resection of $\mathrm{HCC}$ is surveyed by regular ultrasonography or contrast computed tomography scan every 3 months in most centers in order to detect early recurrence that can be treated effectively by re-resection, ablation, or transarterial chemoembolization.

Cucchetti et al. ${ }^{9}$ suggested that strict surveillance should be applied for the first 2 years, but in those with no recurrence after 2 years and low risk for late recurrence, a less stringent surveillance scheme similar to that of surveillance for HCC in cirrhotic patients (i.e., 6-month surveillance) can be applied. Such a strategy seems to make sense as a cost-effectiveness consideration. However, there are some caveats in advocating such a strategy. First, the stratification of risk factors in this study was based on a small sample size of late recurrence in resected patients and occurrence of HCC in cirrhotic patients. Further studies are needed in larger patient population to define the best stratification of patients according to risk of recurrence of HCC. Furthermore, there are other potential risk factors related to the underlying viral etiology that could be potentially incorporated into the risk-stratification system in future studies. For instance, in hepatitis B virus related HCC, recent evidence suggested that patients with hepatitis $\mathrm{E}$ antigen or high hepatitis B virus DNA level are at higher risk of recurrence after resection of HCC. ${ }^{10,11}$ Second, patients who have undergone resection of HCC may have more restricted options of treatment, particularly re-resection, because of the previous partial hepatectomy. It may be of particular advantage to diagnose recurrent tumor earlier in patients who have already had hepatic resection so that less invasive local ablation can be applied. Third, it has to be borne in mind that the stratification of early and late recurrence at 2 years after resection is somewhat arbitrary. While risk factor analysis of early and late recurrence provides insight into the likely mechanism and risk of recurrence in a patient population, it may not specifically predict the risk of recurrence in a patient. For instance, in a patient with a large tumor with microvascular invasion who has no recurrence within the first 2 years after resection, the risk of recurrence due to an occult microscopic metastasis beyond 2 years remains substantial even if the patient's underlying liver condition is at low risk of developing a late multicentric recurrence. It may be prudent to advise more stringent surveillance in such a patient even after 2 years. Currently, the biological behavior of HCC remains largely unpredictable. Before any effective adjuvant therapy is available, early detection and effective treatment of recurrence is the most practical way to prolong patient survival. The cost-effectiveness and the survival benefit of different intervals of postresection surveillance for HCC need to be studied in greater detail in this specific setting before making a recommendation.

The differentiation of early and late recurrence may also have implications on strategies in adjuvant therapy to prevent recurrence. To date, no adjuvant therapies have been conclusively demonstrated to prevent recurrence. However, active clinical trials are being conducted to use new drug therapies to prevent recurrence. One approach is to use molecular targeting drugs that aim at inhibition of tumor cell proliferation, invasion, or angiogenesis. ${ }^{12}$ For such an approach that aims at prevention of growth of micrometastasis from the initial tumor, the endpoint of the trial should be prevention of early recurrence within the first 2 years. In contrast, adjuvant strategies that aim at modulation of the underlying liver condition should target prevention of late recurrence as the end point. For hepatitis virus related HCC, antiviral therapy may be able to reduce hepatitis activity in the cirrhotic liver and decrease the chance of multicentric development of HCC. A randomized trial of lamivudine versus placebo in cirrhotic patient population has shown that lamivudine reduces the risk of development of HCC. ${ }^{13}$ The effect of antiviral therapy on incidence of multicentric recurrence after resection of hepatitis B virus related HCC is certainly worthwhile to study. For hepatitis $\mathrm{C}$ virus related $\mathrm{HCC}$, there is some evidence that interferon may reduce late recurrence after resection of HCC in a randomized controlled trial, though this needs to be verified in further studies. ${ }^{14}$

Finally, the differentiation of the mechanisms of early recurrence and late recurrence may have an important implication on treatment of recurrence, especially in the consideration of salvage transplantation for recurrence. ${ }^{15,16}$ Early recurrence is more likely related to micrometastasis from the initial tumor and may be a less favorable candidate for salvage transplantation, especially for those patients with multinodular recurrence representing widespread micrometastases. On the other hand, a patient with a small late multicentric recurrence after resection of HCC is a more favorable candidate for salvage transplantation, with prognosis probably similar to that of primary 
transplantation for a newly diagnosed small HCC. For cirrhotic patients with high risk of late recurrence as demonstrated by risk stratification and low risk for early recurrence from micrometastasis based on the histological analysis of the resected tumor, it may also be reasonable to consider liver transplantation prior to the development of recurrence. It remains debatable which strategy of salvage transplantation is better, but to some extent this depends on local availability of liver grafts.

In conclusion, risk factor studies of early and late recurrence have provided significant evidence of different mechanisms of early and late recurrences after resection of HCC. The additional knowledge of risk of late recurrence after resection of $\mathrm{HCC}$ in cirrhotic patients compared with risk of occurrence of $\mathrm{HCC}$ in general cirrhotic patients sheds insight into a new strategy of surveillance for recurrence based on risk of late recurrence. The differentiation of early and late recurrences also has important implications on prevention and treatment of recurrences, which are the most important areas of study to improve the long-term prognosis after resection of HCC.

\section{REFERENCES}

1. Poon RT, Fan ST, Wong J. Risk factors, prevention and management of recurrence after resection of hepatocellular carcinoma. Ann Surg 2000;232:10-24.

2. Chan AC, Poon RT, Ng KK, Lo CM, Fan ST, Wong J. Changing paradigm in the management of hepatocellular carcinoma improves the survival benefit of early detection by screening. Ann Surg 2008;247:666-73.

3. Poon RT, Fan ST, Lo CM, Ng IO, Liu CL, Lam CM, et al. Improving survival results after resection of hepatocellular carcinoma: a prospective study of 377 patients over 10 years. Ann Surg 2001;234:63-70.

4. Chen PJ, Chen DS, Lay MY, Chang MH, Huang GT, Yang PM, et al. Clonal origin of recurrent hepatocellular carcinomas. Gastroenterology 1989;96: 527-9.

5. Ng IO, Guan XY, Poon RT, Fan ST, Lee JM. Determination of the molecular relationship between multiple tumour nodules in hepatocellular carcinoma differentiates multicentric origin from intrahepatic metastasis. J Pathol 2003;199:345-53.

6. Poon RT, Fan ST, Ng IO, Lo CM, Liu CL, Wong J. Different risk factors and prognosis for early and late intrahepatic recurrence after resection of hepatocellular carcinoma. Cancer 2000;89:5007.

7. Imamura H, Matsuyama Y, Tanaka E, Ohkubo T, Hasegawa K, Miyagawa S, et al. Risk factors contributing to early and late phase intrahepatic recurrence of hepatocellular carcinoma after hepatectomy. J Hepatol 2003;38:200-7.

8. Portolani N, Coniglio A, Ghidoni S, Giovanelli M, Benetti A, Tiberio GA, et al. Early and late recurrence after liver resection for hepatocellular carcinoma: prognostic and therapeutic implications. Ann Surg 2006;243:229-35.

9. Cucchetti A, Piscaglia F, Caturelli E, Benvegnu L, Vivarelli M,Ercolani G, et al. Comparison of recurrence of hepatocellular carcinoma after resection in patients with cirrhosis to its occurrence in a surveilled cirrhotic population. Ann Surg Oncol 2009;2:413-22.

10. Sun HC, Zhang W, Qin LX, Zhang BH, Ye QH, Wang L, et al. Positive serum hepatitis B e antigen is associated with higher risk of early recurrence and poorer survival in patients after curative resection of hepatitis B-related hepatocellular carcinoma. J Hepatol 2007;47:684-90.

11. Hung IF, Poon RT, Lai CL, Fung J, Fan ST, Yuen MF. Recurrence of hepatitis B-related hepatocellular carcinoma is associated with high viral load at the time of resection. Am J Gastroenterol 2008;103:1663-73.

12. Pang RW, Poon RT. From molecular biology to targeted therapies for hepatocellular carcinoma: the future is now. Oncology 2007;72 Suppl 1:30-44.

13. Liaw YF, Sung JJ, Chow WC, Farrell G, Lee CZ, Yuen H, et al. Lamivudine for patients with chronic hepatitis B and advanced liver disease. $N$ Engl J Med 2004;351:1521-31.

14. Mazzaferro V, Romito R, Schiavo M, Mariani L, Camerini T, Bhoori S, et al. Prevention of hepatocellular carcinoma recurrence with alpha-interferon after liver resection in $\mathrm{HCV}$ cirrhosis. Hepatology 2006;44:1543-54.

15. Poon RT, Fan ST, Lo CM, Liu CL, Wong J. Long-term survival and pattern of recurrence after resection of small hepatocellular carcinoma in patients with preserved liver function: implications for a strategy of salvage transplantation. Ann Surg 2002;235: 373-82.

16. Hwang S, Lee SG, Moon DB, Ahn CS, Kim KH, Lee YJ, et al. Salvage living donor liver transplantation after prior liver resection for hepatocellular carcinoma. Liver Transpl 2007;13:741-6. 